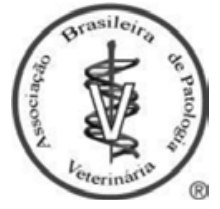

Diagnostic Exercise

From The Latin Comparative Pathology Group*

\title{
Epitheliotropic lymphoma-induced disseminated ulcerative dermatitis in a dog
}

Contributors:

Vanessa J. Oakes*1, Francisco R. Carvallo ${ }^{1}$.

${ }^{1}$ DACVP, Department of Biomedical Sciences and Pathobiology, Virginia-Maryland College of Veterinary Medicine,

Virginia Tech, Blacksburg, VA. USA.

*Corresponding author: E-mail: voakes@vt.edu

\section{Clinical History:}

A young adult, spayed female dog was presented for necropsy by animal control, with concerns for abuse in the form of chemical burns.

\section{Necropsy and Microscopic Findings:}

There was marked alopecia and coalescing ulceration affecting approximately $60 \%$ of the integument, roughly evenly distributed over the body. Affected areas included the mucocutaneous junctions, the palmar and plantar surfaces of the limbs, the paw pads, and the nasal planum. Ulcers measured up to $1 \mathrm{~cm}$ in diameter, frequently coalesced, and varied in stage of development; some were prominent, dark red, and shiny with roughened borders and others contained pink granulation tissue. In areas of alopecia without ulceration, the skin was smooth and glistening. The nails were variably sloughed; some digits possessed a bare quick and some had nails up to $3 \mathrm{~cm}$ long, but which were separating from the underlying quick. The dog was in adequate body condition.

\section{Follow-up questions:}

- Morphologic diagnosis;

- Additional diagnostic tests

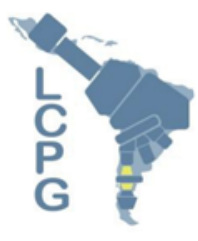

*The Diagnostic Exercises are an initiative of the Latin Comparative Pathology Group (LCPG), the Latin American subdivision of The Davis-Thompson Foundation and published in cooperation with the Brazilian Journal of Veterinary Pathology.

Editor-in-chief for this Diagnostic Exercise: Claudio Barros Associate Editor for this Diagnostic Exercise: Ingeborg Langohr.

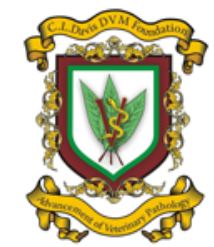


Braz J Vet Pathol, 2021, 14(1), 75 - 79

\section{Gross Images:}

DOI: 10.24070/bjvp.1983-0246.v14i1p75-79

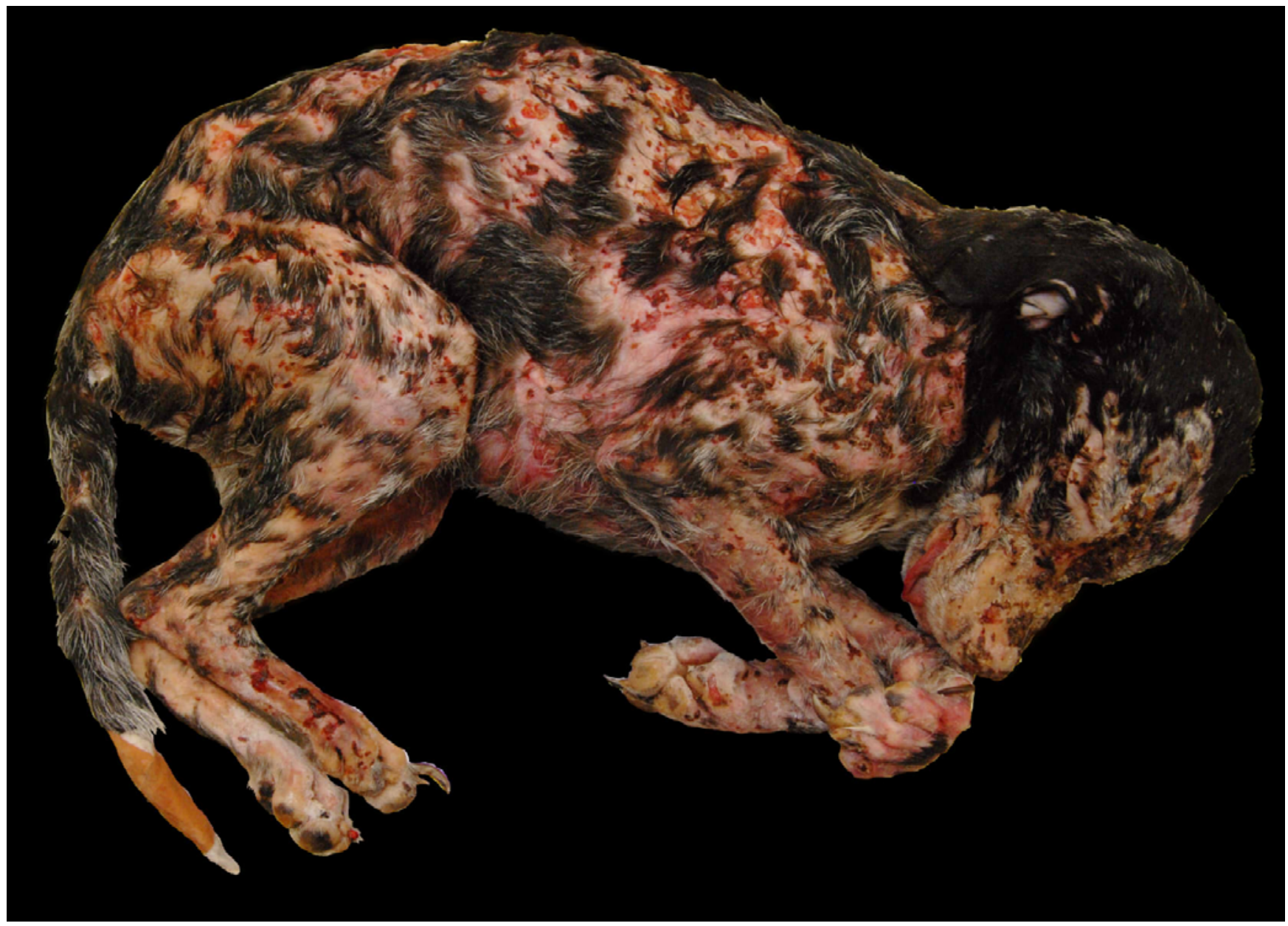

Figure 1

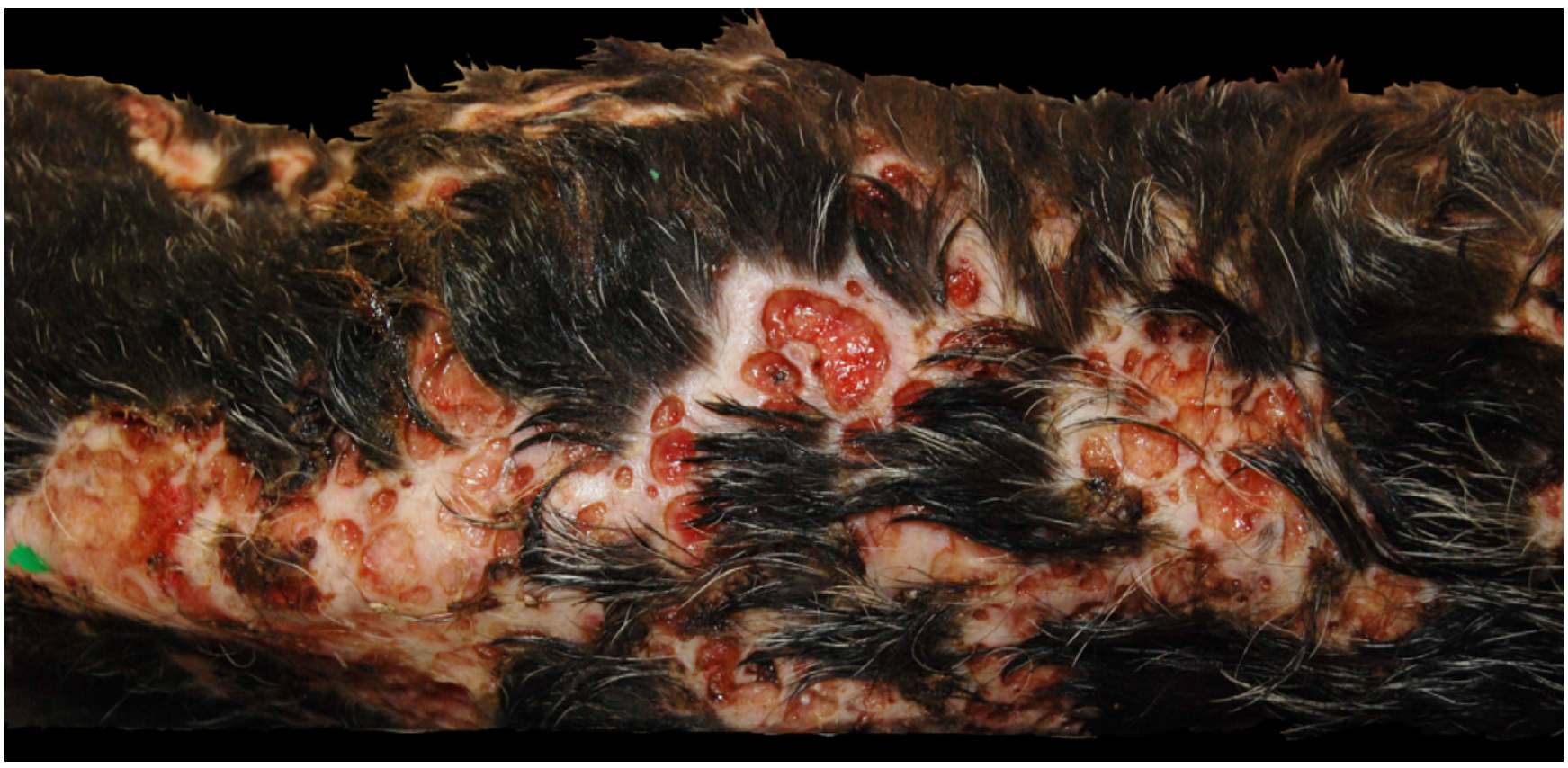

Figure 2 


\section{Microscopic Images:}
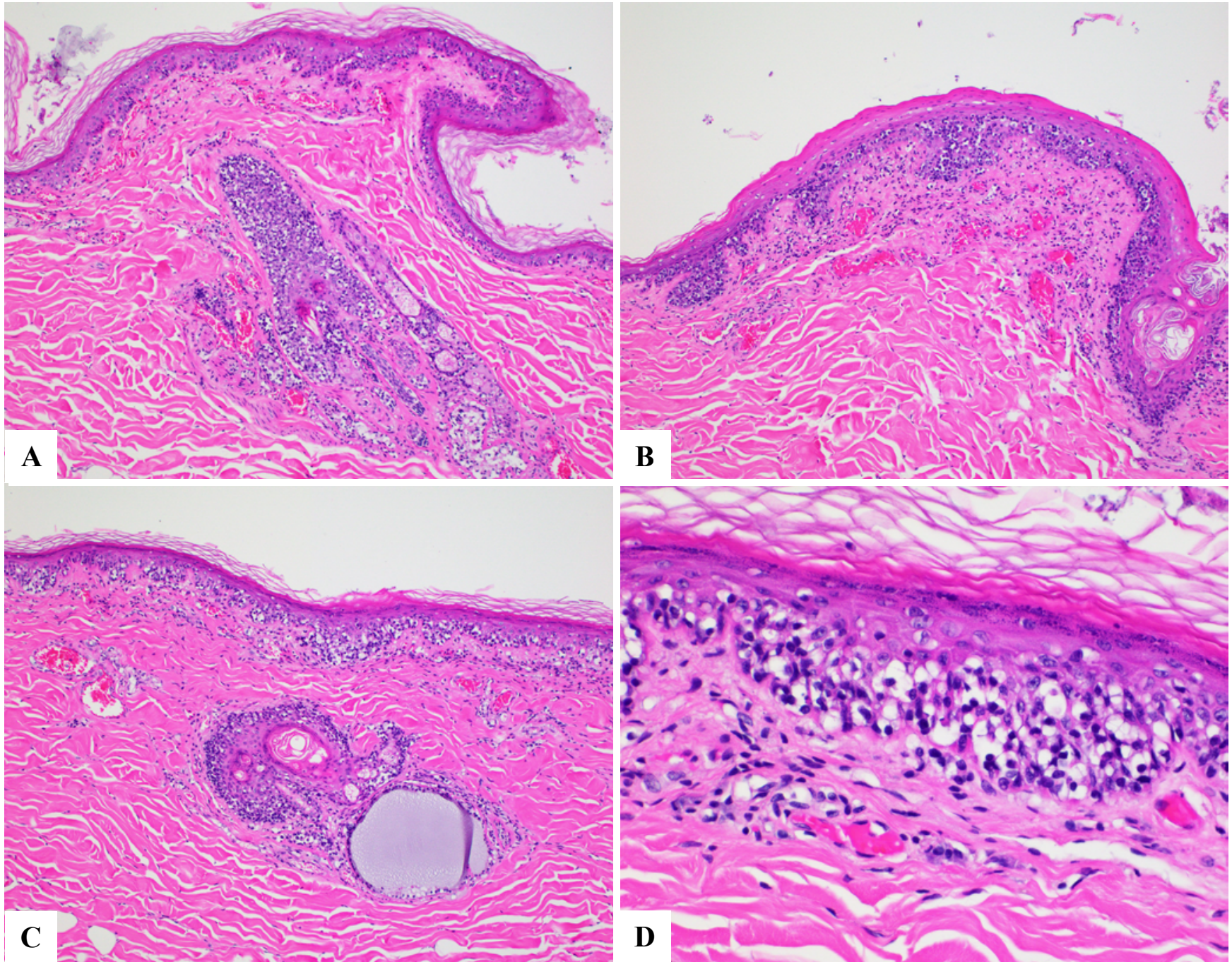

Figure 3. (A) Lymphocytes invade the epidermis, including the lining of hair follicles and adnexal structures. (B) Within the superficial dermis there is a pleomorphic population of lymphocytes mixed with collagen and increased areas of neovascularization. (C) H\&E, 40x. (D) H\&E 400x 


\section{Histologic findings:}

DOI: $10.24070 /$ bjvp.1983-0246.v14i1p75-79
Haired skin. A non-encapsulated, moderately cellular neoplasm is confined to the epidermis, composed of small lymphocytes within the deep epidermal, and follicular and adnexal glandular epithelium. Neoplastic lymphocytes have scant amounts of eosinophilic cytoplasm with distinct cell margins. The nuclei are round and typically hyperchromatic, although occasionally nuclei are open with marginated chromatin and a single, punctate nucleolus. Anisocytosis and anisokaryosis are minimal. No mitotic figures are seen in twelve, $400 \mathrm{x}$ fields (the mitotic count is 0 in $2.37 \mathrm{~mm}^{\wedge} 2$ ). Neoplastic cells often form dense aggregates within the deep epidermis ("Pautrier's microaggregates" or "Pautrier's microabscesses"), and occasional, individual neoplastic cells extend into the stratum spinosum and, rarely, the stratum granulosum. There is rare, individual keratinocyte necrosis and apoptosis throughout all layers of the epidermis, characterized by individualization of keratinocytes, hypereosinophilic cytoplasm and nuclear condensation. Along the surface of the epidermis, there are increased amounts of anuclear, lamellar keratin, occasionally admixed with small numbers of mixed bacterial colonies. The superficial dermis diffusely contains loose, occasionally coalescing aggregates of lymphocytes and plasma cells, sometimes surrounding areas of neovascularization. There are multiple, large foci of complete ulceration of the epidermis, with replacement by necrotic cellular debris and large numbers of bacterial colonies. The underlying dermal collagen is diffusely hypereosinophilic with hyalinization of collagen bands (collagenolysis), interspersed with aggregates of lymphocytes and plasma cells, fibrin, and edema.

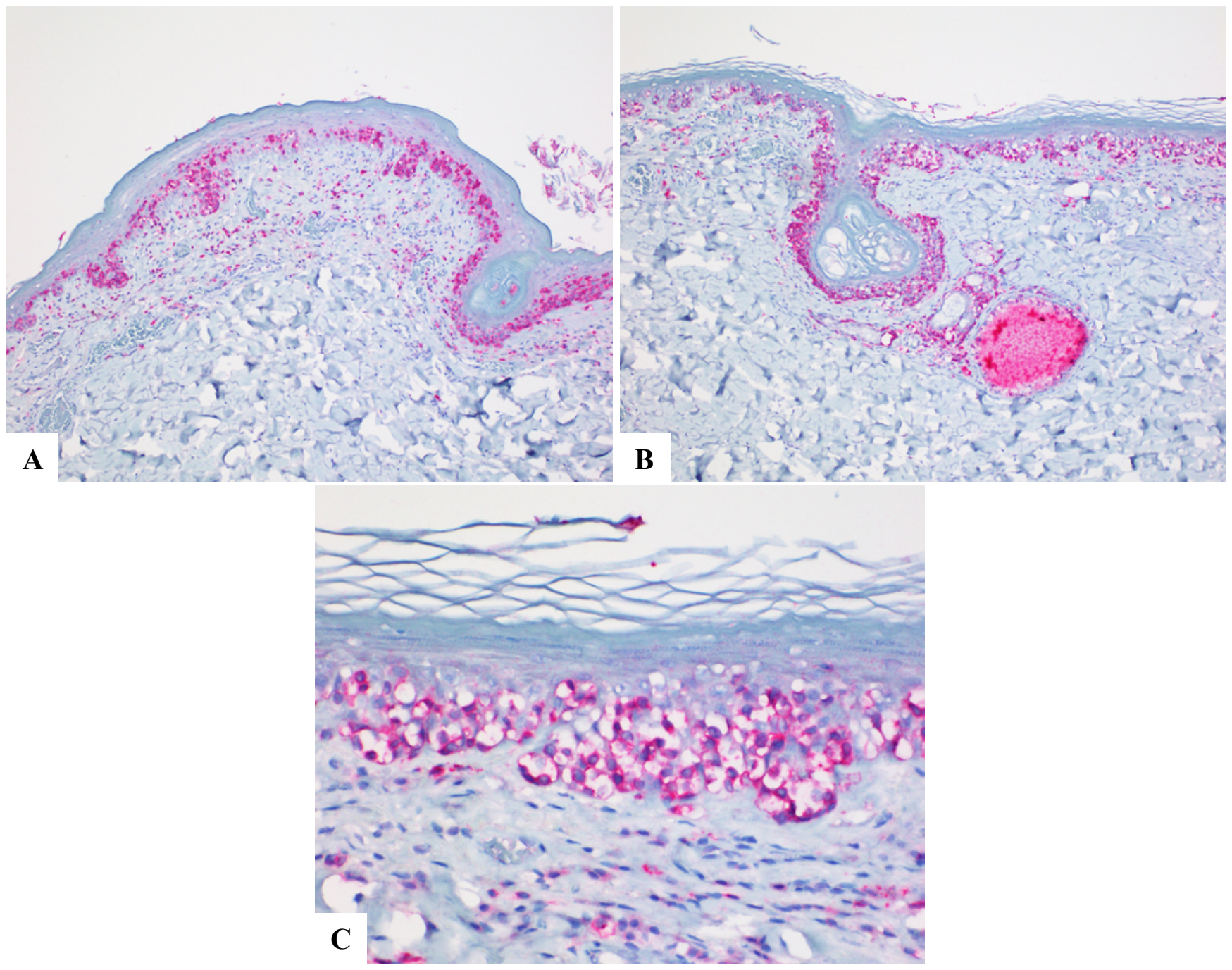

Figure 4. Neoplastic lymphocytes within the epidermis demonstrate diffuse, robust cytoplasmic immunopositivity for CD3.

(A-B) . CD3 IHC. 40x (C) CD3 IHC. 400x. 
Braz J Vet Pathol, 2021, 14(1), 75 - 79

DOI: 10.24070/bjvp.1983-0246.v14i1p75-79

- Diagnosis: Haired skin. Epitheliotropic lymphoma, "pagetoid reticulosis" form, with multifocal, severe ulceration and secondary bacterial dermatitis.

- Additional Diagnostics: Immunohistochemistry for CD3: neoplastic lymphocytes demonstrate diffuse, cytoplasmic immunopositivity.

\section{Discussion:}

Canine cutaneous epitheliotropic T-cell lymphoma (CETL) is a rare neoplastic condition in the skin of dogs, with cutaneous lymphoma altogether (both epitheliotropic and non-epitheliotropic) representing $1 \%$ of all cutaneous neoplasms in dogs (1). Epitheliotropic lymphoma refers to a subcategory of cutaneous lymphoma with distinct tropism of neoplastic lymphocytes for the epidermis and adnexal structures $(3,2)$. There are several subforms of CETL: mycosis fungoides, pagetoid reticulosis, and Sézary syndrome. These classifications derive from the human medical literature and, in practice, there is often overlap of the different types. In veterinary medicine, all forms of CETL carry a poor prognosis, with survival rarely extending beyond 2 years $(1,2)$.

The mycosis fungoides (MF) form is so-named due to its historic resemblance to fungal dermatitis, and is itself further classified into two categories, essentially based on clinical progression: classic and d'emblée MF. Classic MF progresses clinically through several stages of development, including patch, plaque, and tumor stages (sonamed for the progression of the disease in humans); d'emblée MF consists only of the tumor stage. The histologic lesions are essentially the same in both, and are characterized by the presence of neoplastic lymphocytes diffusely within the epidermis as well as in the dermis. Pigmentary incontinence is a common finding in areas, in which the dermal-epidermal junction is effaced. With disease progression, the neoplastic population expands to efface adnexal structures. The tumor stage is characterized by more blastic lymphocytes than the plaque and patch stages, which have a lesser degree of adnexal effacement and are composed of less pleomorphic lymphocytes (3).

In comparison to the MF form, the pagetoid reticulosis (PR) form of CETL is characterized by neoplastic cells that are constrained to the epidermis and adnexa, such as seen in this case, with no extension of neoplastic cells into the dermis (4). With disease progression, neoplastic lymphocytes can invade beyond the epidermis, however; in these cases, differentiation between the PR and MF forms of CETL is impossible. Due to this, some groups consider the PR form a manifestation of MF. Further, the human literature identifies two sub-types of PR: a localized form (WoringerKolopp PR) and a generalized form (Ketron-Goodman PR) (1). The localized form is considered to be a benign lymphoproliferative disease in many human cases (1).
Sézary syndrome (SS) is histologically identical to the MF form, but with the presence of small lymphocytes containing hyperchromatic, convoluted nuclei (Sézary cells). Additionally, neoplastic cells are found within lymph nodes and circulating within the blood; in some cases, they are within the spleen, liver, lungs, heart, and kidneys as well. In these cases, it is important to differentiate between SS, which is a true epitheliotropic lymphoma, and cases of nonepitheliotropic lymphoma with cutaneous metastasis. In the latter case, neoplastic lymphocytes are typically arranged perivascularly within the dermis, with no obvious tropism for the epithelium (3).

In all subforms of MF, variable numbers of apoptotic keratinocytes are present in all layers of the epidermis, and care must be made to differentiate CETL from autoimmune diseases of the skin.

Gross presentation of CETL varies widely depending on the lesion's distribution, the degree of invasion, and follicular involvement; indeed, it can resemble virtually any other alopecic, ulcerative, or proliferative dermatitis. There was cause for concern of extensive chemical burn for this case, examined at the Virginia-Maryland College of Veterinary Medicine.

An etiology of CETL has yet to be identified. A review of 30 cases of CETL did not identify chronic dermatitis as a predisposing factor (2), and there is no evidence of viral involvement in dogs.

\section{References}

1. Fontaine J., Bovens C., Bettenay S., Mueller R.S. 2009. Canine cutaneous epitheliotropic T-cell lymphoma: A review. Vet Comp Oncol 7:1-14.

2. Fontaine J., Heimann M., Day M.J. 2010. Canine cutaneous epitheliotropic T-cell lymphoma: A review of 30 cases. Vet Dermatol 21:267-275.

3. Gross T.L., Ihrke P.J., Walder E.J., Affolter V.K. 2005. Skin Diseases of the Dog and Cat: Clinical and Histopathologic Diagnosis. 2 ed. p. 876-882.

4. Johnson J.A., Patterson J.M. 1981. Canine epidermotropic lymphoproliferative disease resembling pagetoid reticulosis in man. Vet Pathol 18:487-493. 